\title{
Simulation of the Hemodynamic Effects of the Left Atrial Appendage Occlusion in Atrial Fibrillation: Preliminary Results
}

\author{
Nadia D’Alessandro ${ }^{1}$, Alessandro Masci ${ }^{1}$, Alice Andalò ${ }^{1}$, Luca Dedè ${ }^{2}$, Corrado Tomasi ${ }^{3}$, Alfio \\ Quarteroni $^{2}$, Cristiana Corsi ${ }^{1}$ \\ ${ }^{1}$ DEI, Cesena Campus, University of Bologna, Italy \\ ${ }^{2}$ MOX, Polytechnic University of Milan, Milan, Italy \\ ${ }^{3}$ Santa Maria delle Croci Hospital, AUSL della Romagna, Ravenna, Italy ${ }^{2}$
}

\begin{abstract}
Atrial fibrillation $(A F)$ is responsible for $15-18 \%$ of all strokes. In AF patients, the left atrial appendage (LAA) represents the main thrombogenic spot, being the site of 90\% of intracardiac thrombus formation. Therefore, the occlusion of the LAA (LAAO) is a novel strategy for cardioembolic stroke prophylaxis. The aim of this study was the simulation of the fluid dynamics effects of the $L A A O$ in $A F$ patients, by applying two different devices $\left(\right.$ Amulet $^{T M}$ and Watchman $\left.{ }^{T M}\right)$, in order to predict patientspecific hemodynamic changes due to LAAO and to detect the most effective devices in reducing stroke risk as well.
\end{abstract}

\section{Introduction}

In atrial fibrillation (AF) patients, a five-fold increased stroke risk, resulting from thrombus formation, is the most life-threatening condition. In $90 \%$ of cases, the left atrial appendage (LAA) is the site of intracardiac thrombus formation in patients with AF [1]. Left atrial appendage (LAA) is a 2-4 cm hook-shaped projection from the main body of the LA, whose junction with the atrium is well defined by its orifice. In conditions of reduced atrial motility, its complex morphology predisposes blood stasis, clot formation and embolism [2]. Therefore, a novel nonpharmacological strategy for cardioembolic stroke prophylaxis in patients with $\mathrm{AF}$ is represented by percutaneous, transcatheter LAA occlusion (LAAO) based on endocardial devices. Recent trials have shown that LAAO is effective and not inferior to oral therapy in stroke prevention [3-5]. However, the device implant might be associated with major adverse events, so the utmost attention must be paid while balancing benefits and risks linked to this procedure [6]. Also, the choice of the most appropriate device assumes primary importance, because of the large intra-subject anatomical variation of LAA.

Computational fluid dynamics (CFD) represents a valuable non-invasive approach to estimate physically significant hemodynamic parameters in a complex fluid dynamics system. It might provide a helpful in silico simulation of the blood flow patterns within the LA and
LAA by using 3D patient-specific models. Prior CFD studies have analysed the hemodynamics in different LAAs to identify potential relationships between the morphology and the risk of thrombotic events [2,7-9]. As of today, few studies are focused on the hemodynamic changes in LA pre- and post-LAAO [10,11]. Yet, the hemodynamic effects depending on the closure morphology at the ostium have not been investigated. Also, in previous works, AF motion condition has been replaced with rigid LA walls, introducing a considerable simplification.

This study's aim was to simulate the fluid dynamics effects of the LAAO in AF patients to predict patientspecific hemodynamic changes caused by the LAAO, by applying the two most popular devices (Amulet ${ }^{\mathrm{TM}}$, Abbott; Watchman ${ }^{\mathrm{TM}}$, Boston Scientific). For this purpose, LAAO was reproduced on 3D LA anatomical models obtained from real clinical data. For each patient, CFD simulations in $\mathrm{AF}$ condition were performed both on the entire atrium model and on the models with LAAO. Significant fluid dynamics indices were determined to evaluate the changes in the flow patterns after the occlusion in relation to the thrombogenic risk.

\section{Methods}

A schematic workflow is shown in Figure 1.

\subsection{Patients Dataset}

The initial dataset consisted of two 3D anatomical models of the LA, extracted from two AF patients CT images with specifically image segmentation algorithms described in [12].

\subsection{LAA Exclusion}

As a first step to generate models with LAAO, we intended to remove LAA from the anatomical models. Therefore, an algorithm implemented in MATLAB which automatically recognized and excluded the LAA was 


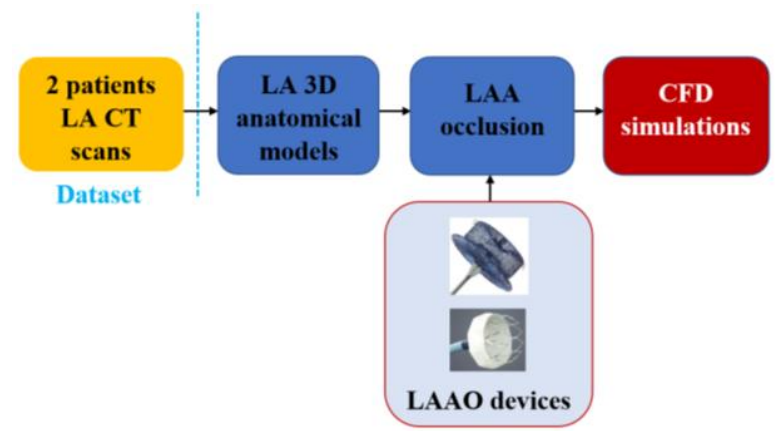

Figure 1. Project workflow.

applied to each mesh. As described in [9], the shape diameter function (SDF) proposed by Shapira et al. [13] was employed. The iso-contours of the SDF map on the LA meshes allowed a threshold-based segmentation in anatomical regions with similar SDF values, identifying pulmonary veins (PVs), atrial chamber and LAA. Based on the anatomical position of the LAA, the algorithm was then able to detect the LAA and to remove it.

\subsection{Closure of LAA ostium}

After removing the LAA, we simulated the occlusion by generating a surface at the orifice. The closing surfaces were created by using MeshLab [14], a 3D mesh processing system. The main geometric features of the devices as well as their fitting in the shape of the LAA ostium were considered. The Amulet ${ }^{\mathrm{TM}}$ nitinol disc sealing the LAA orifice was emulated with a flat planar closure. The screened Poisson surface reconstruction [15] algorithm was instead used to reproduce the umbrellashaped structure of the Watchman ${ }^{\mathrm{TM}}$ device. For each patient, we obtained a final set of three LA anatomical models: the model with LAA; (2) the LAAO model with Amulet $^{\mathrm{TM}}$; (3) the LAAO model with Watchman ${ }^{\mathrm{TM}}$ (Figure 2).

\subsection{The Computational Fluid Dynamic Model and Numerical Simulation}

These anatomical models represented the computational domain for the CFD model described in Masci et al. [12], which was used to simulate atrial fluid dynamics in AF condition. The blood flow was modelled as an incompressible fluid governed by the Navier-Stokes equations. The atrial contraction in $\mathrm{AF}$ condition was simulated by applying independently to each vertex of the anatomical LA model a random displacement with small amplitude $(0.1 \mathrm{~mm})$ and frequency $(4 \mathrm{~Hz})$ fixed at a typical value for AF. The numerical discretization of the CFD model was implemented in LifeV [14] library. Each simulation was run for five cardiac cycles to remove the
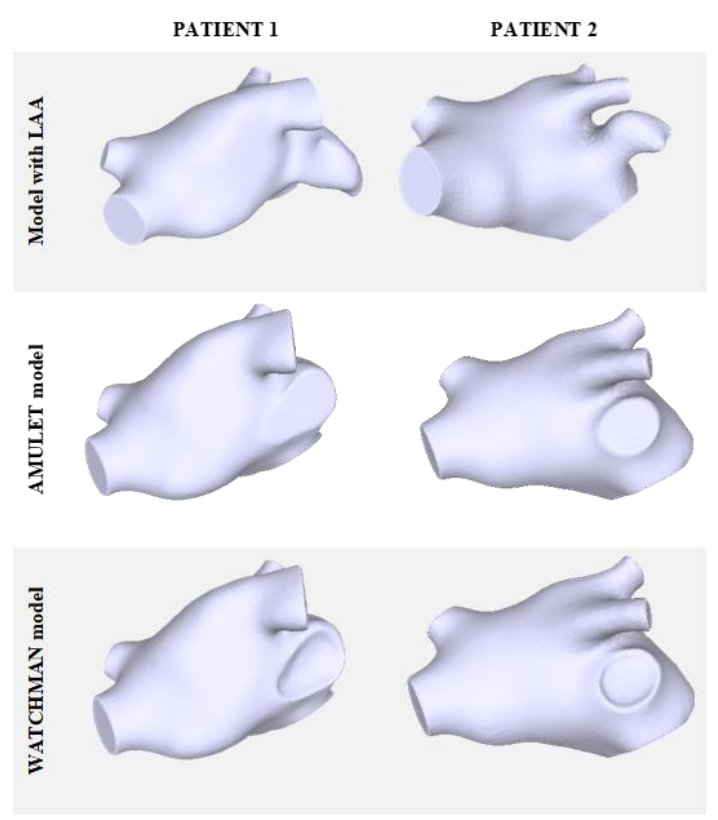

Figure 2. LA models with the LAA (upper panels) and after LAAO with Amulet ${ }^{\mathrm{TM}}$ and Watchman ${ }^{\mathrm{TM}}$ devices (mid and bottom panels).

dependence on initial conditions. Results from the last cardiac cycle are reported. For each simulation, fluid dynamics parameters such as velocity, vortex structures, LAA ostium velocity and LA blood stasis were computed. For the visualization of the vortex structures the Qcriterion was employed.

\section{Results and Discussion}

\subsection{Velocity Analysis}

As for patient 1 , fluid dynamics simulations with the LAA showed different spatial distribution and higher velocity peaks compared to LAAO. Velocity vectors showed more disorganized and turbulent patterns in the pre-LAAO case, while a more directed mitral outflow was observed after the occlusion. Decrease in velocity was detectable in the most distal areas of the LA, at the anterosuperior and septal walls and at PVs inflows. However, velocities at the mitral valve and at the LAA ostium were higher after LAAO. Maximum velocities were recorded at mitral valve (MV) during ventricular diastole $(72 \mathrm{~cm} / \mathrm{s}$ Amulet; $69 \mathrm{~cm} / \mathrm{s}$ Watchman), compared to $60 \mathrm{~cm} / \mathrm{s}$ in the model with LAA. Before the occlusion, an emptying of the LAA was never observed and velocity did not exceed 10 $\mathrm{cm} / \mathrm{s}$. On the contrary, in LAAO models, incremented velocities were recorded throughout the cardiac cycle near the ostium with a noticeable peak speed of $64 \mathrm{~cm} / \mathrm{s}$. A more disorganized fluid pattern was observed for the Watchman ${ }^{\mathrm{TM}}$ LAAO device, probably due to its concave 
shape that causes variations in the fluid streamlines direction. On average, velocities were slightly higher in the Watchman model than in the Amulet.

In patient 2 models, more similar blow flow patterns were identified in pre- and post-LAAO cases. In the entire atrium model, the LAA was poorly involved in the blood washout, as suggested by the near-zero speeds. The removal of LAA produced a slight reduction in speed at PVs and at LA inferior-septal wall. Flow to the mitral valve reached comparable rates in the three models $(50 \mathrm{~cm} / \mathrm{s}$ in both LAAO models; $46 \mathrm{~cm} / \mathrm{s}$ in model with LAA). During atrial systole, a difference in blood flow pattern was noticeable (Figure 3(a)). Indeed, in pre-occlusion LA, an isolated backward oriented peak of velocity $(46 \mathrm{~cm} / \mathrm{s})$ was detected under the left PVs, while in the center of the atrial chamber velocities were much lower. On the contrary, in both LAAO models, a larger distributed peak is observed in the center of the atrial chamber, suggesting an improved washout. Importantly, the highest velocity vectors $(50 \mathrm{~cm} / \mathrm{s}$ in Amulet; $50 \mathrm{~cm} / \mathrm{s}$ in Watchman) appeared towards the MV. Overall, very similar behaviors were observed between the two types of closure.

\subsection{Vortex Structures Analysis}

In general, the presence of vortices could promote a more effective washout effect and avoid blood stagnation. On the contrary, a decrease in their number, size and speed could lead to thrombus formation.

In both patients, vortex structures were almost absent within the LAA and mostly in its apical part throughout the entire cardiac cycle, thus revealing an inadequate blood washout of the zone.

As for patient 1, after the closure, vortices were less numerous in LA, but bigger in size. Higher velocity vortices were located at $\mathrm{MV}$ and in proximity of the LAAO. In the LAAO models, vortex structures were more localized in the centre of the chamber. The model with the Watchman showed a slightly higher number of vortices than the Amulet.

In accordance with the velocity analysis for patient 2, we observed less appreciable changes due to the LAAO. After the LAAO, more vortices were found near the closure. Generally, vortex structures minimally decreased in number in the LA. We noticed some differences in vortices localization and speed distribution, with lower velocity at PVs in ventricular diastole (Figure 3(b)) but higher velocity near the MV during atrial systole.

\subsection{LAA Ostium Velocity}

Studies in literature [15] have shown that speeds below $40 \mathrm{~cm} / \mathrm{s}$ in LA are associated with a higher risk of stroke, while speeds below $20 \mathrm{~cm} / \mathrm{s}$ are associated with the identification of thrombus.
In patient 1 model with the LAA, velocity values at the ostium fluctuated between $8 \mathrm{~cm} / \mathrm{s}$ and $19 \mathrm{~cm} / \mathrm{s}$ throughout the cardiac cycle, highlighting a potential thrombogenic spot. Vice versa, in both LAAO models much increased average velocity was registered $(13.0 \mathrm{~cm} / \mathrm{s}$ model with LAA; $25.2 \mathrm{~cm} / \mathrm{s}$ Amulet; $31.1 \mathrm{~cm} / \mathrm{s}$ Watchman), with peaks over $50 \mathrm{~cm} / \mathrm{s}$, suggesting a reduced stroke risk.

The effects of LAAO in LAA ostium velocity were similar in patient 2 . In the model with the LAA, values ranged from $6 \mathrm{~cm} / \mathrm{s}$ to $15 \mathrm{~cm} / \mathrm{s}$. In LAAO models, average velocity doubled $(10.2 \mathrm{~cm} / \mathrm{s}$ model with LAA; $19.1 \mathrm{~cm} / \mathrm{s}$ Amulet; $20.0 \mathrm{~cm} / \mathrm{s}$ Watchman). In both patients, the Watchman model showed higher average speed.

\subsection{Blood Stasis Quantification}

To quantify blood stasis, each LA was populated by 5000 fluid particles at the beginning of the CFD simulations and the number of particles which remained within the LA at the end of the fifth cardiac cycle (Table 1) was computed. The higher the number of residual particles, the greater the probability of blood stagnation.

In patient 1 , the results showed a small percentage of stasis within the LAA ( $8 \%$ of the total residual particles). Furthermore, it was found that the number of residual particles in the models with LAAO (4.1\% Amulet; $3.7 \%$ Watchman) was lower than in the model with LAA $(5.7 \%)$,

In patient $2,5.2 \%$ of the residual particles was found inside LAA. In this case too, the percentage of stasis in the models with LAAO (6.2\% Amulet; 5.3\% Watchman) was lower than in the model with LAA $(7.3 \%)$.

In both cases, a more effective atrial washout in the Watchman configuration was confirmed. These results agreed with the results of velocity, vorticity, and velocity at the LAA ostium.

Table 1. Residual particles in LA after five cardiac cycles.

\begin{tabular}{|c|c|c|c|c|}
\hline & $\begin{array}{c}\text { Cardiac } \\
\text { cycle }\end{array}$ & $\begin{array}{l}\text { Model with } \\
\text { LAA }\end{array}$ & $\begin{array}{c}\text { Amulet } \\
\text { model }\end{array}$ & $\begin{array}{c}\text { Watchman } \\
\text { model }\end{array}$ \\
\hline$=$ & 0 & 5000 & 5000 & 5000 \\
\hline صี & 5 & $\begin{array}{c}285 \\
(23 \text { in LAA) }\end{array}$ & 205 & 183 \\
\hline$\stackrel{\sim}{\rightleftharpoons}$ & 0 & 5000 & 5000 & 5000 \\
\hline 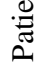 & 5 & $\begin{array}{c}365 \\
\text { (19 in LAA) }\end{array}$ & 310 & 265 \\
\hline
\end{tabular}

\section{Conclusions}

In this study a workflow for simulating the fluid dynamics effects of LAAO in AF was tested. The study could contribute to understand the fluid dynamics conditions leading to thrombogenesis and to identify the 

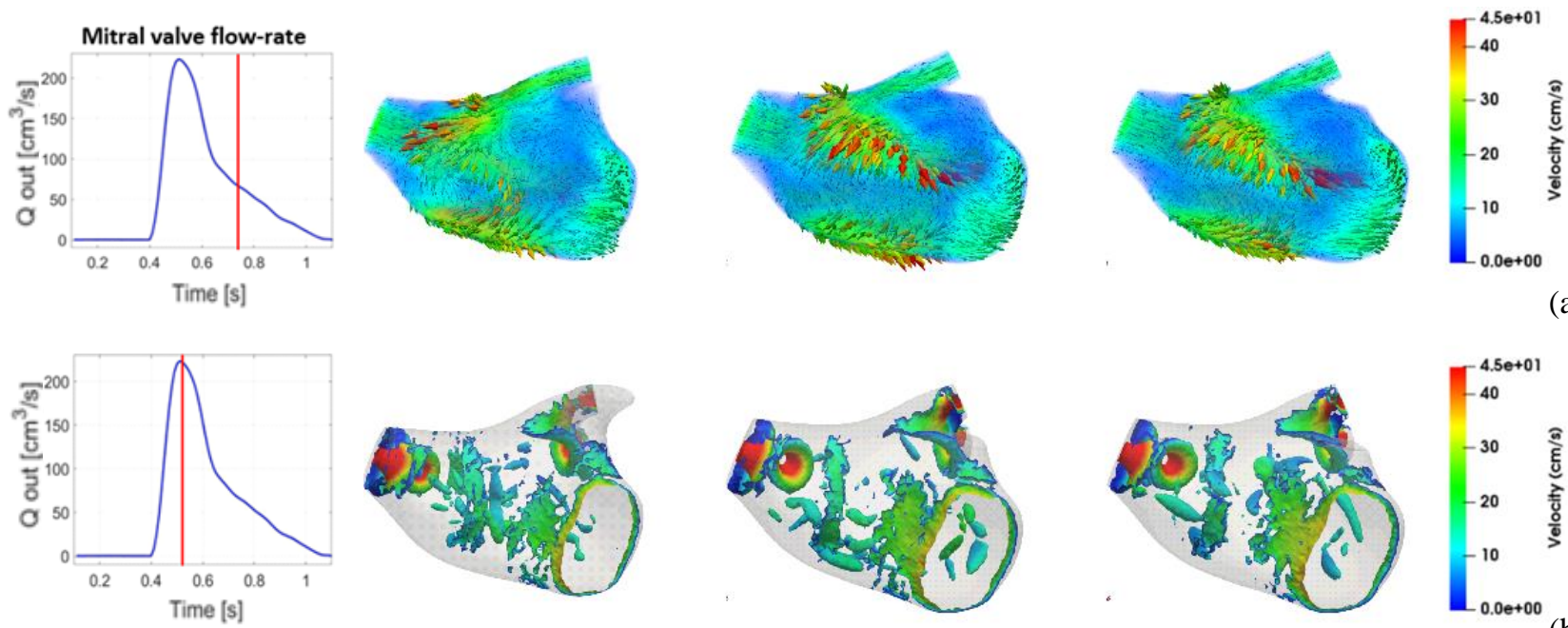

(a)

Figure 3. First column reports the computed flow rate at the MV. Each row corresponds to a different time in the cardiac cycle, denoted by the vertical red marker: atrial systole (upper panels); ventricular diastole (bottom panels). Results of (a) velocity (upper panels) and (b) vorticity (bottom panels) for patient 2 model with LAA (left) and after LAAO with Amulet ${ }^{\mathrm{TM}}$ (mid) and Watchman ${ }^{\mathrm{TM}}$ (right) are shown.

most effective devices in reducing the stroke risk for patient-specific morphologies of LA. Our preliminary results suggested a more effective blood washout after LAAO and, consequently, a lower risk of blood clot formation. Also, a slightly improved washout effect is revealed when the Watchman ${ }^{\mathrm{TM}}$ device is implanted. These results need confirmation in a larger population.

\section{References}

[1] Al-Saady N.M., Obel O.A., Camm A.J. Left atrial appendage: structure, function, and role in thromboembolism. Heart 1999; 82(5):547-54.

[2] Bosi G. M., Cook A., Rai R., Menezes L. J. Schievano S., Torii R., Burriesci G., Computational Fluid Dynamic Analysis of the Left Atrial Appendage to Predict Thrombosis Risk. Front. Cardiovasc. Med. 2018; 5:34.

[3] Osmancik P., Tousek P., Herman D., et al. Interventional left atrial appendage closure vs novel anticoagulation agents in patients with atrial fibrillation indicated for long-term anticoagulation (PRAGUE-17 study). Am Heart J. 2017;183:108-114.

[4] Holmes Jr. D.R., Kar S., Price M.J., et al. Prospective randomized evaluation of the Watchman Left Atrial Appendage Closure device in patients with atrial fibrillation versus long-term warfarin therapy: the PREVAIL trial. J Am Coll Cardiol 2014; 64:1-12.

[5] Fountain R.B., Holmes D.R., Chandrasekaran K., et al. The PROTECT AF (WATCHMAN Left Atrial Appendage System for Embolic PROTECTion in Patients with Atrial Fibrillation) trial. Am Heart J. 2006;151(5):956-961.

[6] Aryana A., d'Avila A. Incomplete Closure of the Left Atrial Appendage: Implication and Management. Curr Cardiol Rep 2016; 18(9):82.

[7] Di Biase L., Santangeli P., Anselmino M., et al. Does the Left Atrial Appendage Morphology Correlate with the Risk of Stroke in Patients with Atrial Fibrillation? Results From a Multicenter Study. Journal of the American College of Cardiology 2012;60(6):531-538.

[8] Jeong W.K., Choi J.H., Son J.P., et al. Volume and Morphology of Left Atrial Appendage as Determinants of Stroke Subtype in Patients with Atrial Fibrillation. Heart Rhythm 2016;13(4):820-827.

[9] Masci A., Barone L., Dedé L., et al. The impact of left atrium appendage morphology on stroke risk assessment in atrial fibrillation: a computational fluid dynamics study. Front. Physiol. 2019;9:1938.

[10] Aguado A.M., Olivares A.L., Yagüe C., et al. In silico Optimization of Left Atrial Appendage Occluder Implantation Using Interactive and Modeling Tools. Front Physiol. 2019;10:237.

[11] Dongjie J., Byunghwan J., Hyung-Bok P., et al. ImageBased Flow Simulations of Pre- and Post-left Atrial Appendage Closure in the Left Atrium. Cardiovascular Engineering and Technology, 2019; 10(2):225-241.

[12] Masci A., Alessandrini M., Forti D., et al. A proof of concept for computational fluid dynamic analysis of the left atrium in atrial fibrillation on a patient-specific basis, J. Biomech. Eng., 2020; 142(1): 011002.

[13] Shapira, L., Shamir, A. \& Cohen-Or, D. Consistent mesh partitioning and skeletonisation using the shape diameter function. Visual Comput 24, 2008; 249-259.

[14] Malossi, C., and Deparis, S. LifeV Development Guidelines. 2009.

[15] Beigel R., Cercek B., Luo H. et al. Noninvasive evaluation of right atrial pressure. $\mathrm{J}$ Am Soc Echocardiogr 2013;26:1033-42.

Nadia D'Alessandro

Department of Electric, Electronic and Information Engineering Guglielmo Marconi DEI, University of Bologna,

Via dell'Università 50, 47521 - Cesena (FC)

nadia.dalessandro@studio.unibo.it 